\title{
Smart Routing with Segmental Routing Mechanism for Wireless Mesh Network (WMN)
}

\author{
Manmeet Kaur, Surinder Singh, Preeti Bansal, Gaurav Kumar
}

\begin{abstract}
The wireless mesh networks (WMN) are the growing mediums of connectivity for the purpose of internet or intranet connectivity. The routing among the WMN becomes a challenging task with the rise in the number of nodes across the network and the larger data volumes. In this paper, the work has been carried out on the growing WMNs with the dynamic number of nodes. The node availability aware neighbor formation and status tracking mechanism has been applied in this scheme in order to keep the network updated about the working nodes and to eliminate the non-functional nodes from the connected mesh network. The neighbor query process is utilized for the path building towards the base stations (BTS) or Sink node in the given WMN networks. Additionally; the routing information is collected from all of the neighboring nodes towards the destination paths using the desired mechanism for the path discovery under the segmental routing mechanism for WMN, which has been created with the capability of handling the network path failures dynamically in the local domain of the given network zone. The performance of the proposed model has been analyzed in the form of energy consumption, end-to-end delay and detailed energy \& packet based analysis
\end{abstract}

Index Terms: Segmental routing, WMN routing, Mesh networking, Routerecovery

\section{INTRODUCTION}

The wireless mesh network (WMN) is a form of wireless networks, and are known as the complex wireless topologies, which are constructed by combining the multiple network architecture together in order to create a flexible and multi-scale wireless network to provide the maximized connectivity. The WMN architectures or environments includes the various wireless standards altogether such as WiFi, WiMax, Mobile ad-hoc networks (MANETs), Cellular Networks, etc. These networks offer the voice connectivity, data sharing services and several other integrated services to its users. [7]

Revised Manuscript Received on June 15, 2019.

Manmeet Kaur, Department of Electronics and Communication Engineering, CEC Landran, Mohali, Punjab, India

Surinder Singh, Department of Electronics and Communication Engineering, CEC Landran, Mohali, Punjab, India

Preeti Bansal, Department of Electronics and Communication Engineering, CEC Landran, Mohali, Punjab, India

Gaurav Kumar, Department of Electronics and Communication Engineering, CEC Landran, Mohali, Punjab, India

The WMNs are known to maximize the network coverage and inter-connect the largest number of users, as they can accommodate the users from multiple networks on a single platform. The inter-cellular network connectivity such as inter-connection between Bharti Airtel, BSNL, Reliance Jio, Vodafone or Idea users with each other, defines the real power of this network standard. A typical WMN offers the highly dynamic and cost effective connectivity over the larger geographical coverage area in order to cover the millions of users as it's shown in figure 1. The WMNs can be divided into the multiple segments, which can be distinguished by network type, geographic area, etc. The WMN manages the permanent as well as temporary network paths, which are manages the backbone paths (primary paths to connect different segments).

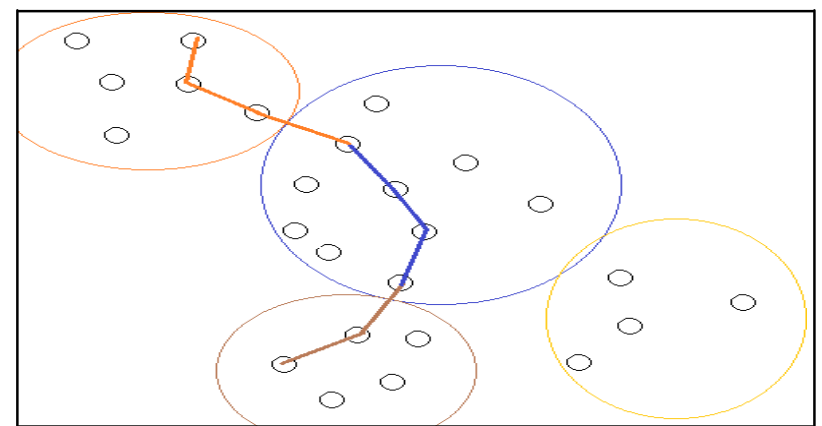

Fig. 1: A typical Wireless Mesh Network

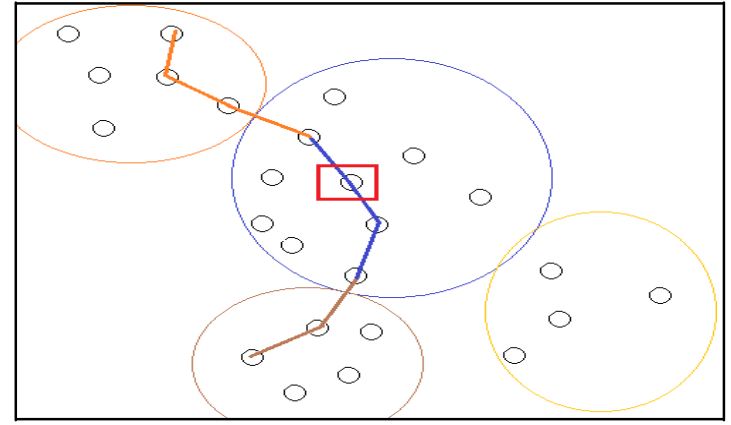

Fig. 2: WMN showing the node failure with red square

Figure 2: WMN showing the node failure with red square the smaller paths are discovered among the different network segments in order to share the data between two nodes from different segments. In this paper, a new mechanism has been proposed for the routing among the wireless mesh networks, where the routing has been shifted in the local domain in the segment scope. [8]The segment scope based local domain network management is the procedure offered in the proposed model, where the network path between the 
two nodes from different segments in managed smartly and quickly.

The smaller paths are discovered among the different network segments in order to share the data between two nodes from different segments. In this paper, a new mechanism has been proposed for the routing among the wireless mesh networks, where the routing has been shifted in the local domain in the segment scope [8]. The segment scope based local domain network management is the procedure offered in the proposed model, where the network path between the two nodes from different segments in managed smartly and quickly.

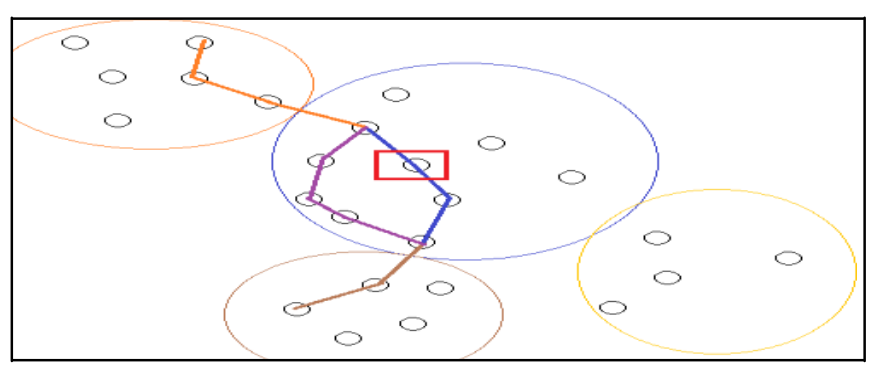

Fig. 3: An alternate path during the node failure

\section{LITERATURE REVIEW}

Al-Saadi, Ahmed et. al. [11] has worked on the Routing Protocol for Heterogeneous Wireless Mesh Networks. This paper proposes a heterogeneous metropolitan area network architecture that combines an IEEE 802.11 wireless mesh network (WMN) with a long-term evolution (LTE) network. Zhou, Anfu et. al. [12] has worked on the Joint Traffic Splitting, Rate Control, Routing, and Scheduling Algorithm for Maximizing Network Utility in Wireless Mesh Networks. In this paper, the authors have focused on exploiting multiple gateways optimally to achieve maximum network utility. Murugeswari, R. [13] has worked on the multi-objective evolutionary algorithm based QoS routing in wireless mesh networks. This paper proposes a new model for routing in WMN by using Modified Non-dominated Sorting Genetic Algorithm-II (MNSGA-II). Sakamoto, Shinji et. al. [14] has worked on the implementation of a New Replacement Method for WMNs. 2014 Delaney, D., Russell Higgs, and G. O'Hare [1] have worked on a stable routing framework for tree-based routing structures in WSNs. The central concept introduces neighborhood heuristics (NHs), a method of combining a sensor's routing metric with those of its neighbors to highlight both the quality of the current route and the quality of the routing options available to the sensor should its current route become unavailable. 2014 Ghadimi, Euhanna et. al. [2] have proposed Opportunistic Routing in Low Duty-Cycled Wireless Mesh networks. In this paper the authors have introduced ORW, a practical opportunistic routing scheme for wireless mesh networks. ORW uses a novel opportunistic routing metric, EDC, which reflects the expected number of duty-cycled wakeups that are required to successfully deliver a packet from source to destination. 2014 Sahin, Dilan et. al. [3] has worked upon QoS differentiation in single-path and multi-path routing for wireless mesh network-based smart grid applications.
Wireless mesh network (WSN) concept places an important role in this modernization process of the power grid with its efficient and low-cost deployment characteristics. 2014 Singh, Dharmendra [4] et. al. has developed an energy efficient source based tree routing with time stamp in WSN. Wireless mesh network most recently used technology. Different routing protocols and topologies are used to transmit data from source to sink. 2012 Kwon, Kiwoong et. al. [5] has worked upon the stateless point to point routing protocol based on shortcut tree routing algorithm for IP-WSN. To address the routing challenges, the authors have proposed the Stateless P2P Routing protocol (SPR) based on shortcut tree routing algorithm which is our previous study.

\section{EXPERIMETNAL DESIGN}

The dynamic route update algorithm has been proposed and designed for the dynamic route updating in the segmental (micro) routing mechanism when operating within the given zone. The inter-zone handovers are treated with the complete route updates in order to remove the chances of bad route origination. The adaptive load balance balancing based new method [6] has been incorporated in this new metric route to find the shortest route with balanced, efficient and higher bandwidth based route selection mechanism to make the delivery process faster and to avoid the congestion and data loss. The route cost calculation for load balancing has been designed on the basis of individual load on the relay node/s, the alternative routes with the minimum load are be also considered to find the alternative route. Among the shortlisted routes using the load as metric, the route with minimum total route cost has been employed to forward the data [9]. This proposed model improvises the utilization in the terms of end to end delay/latency, packet delivery ratio, network/route load and packet Efficiency of the alternative route. The path is observed within the network by using the dijisktra routing algorithm, which is known to discover the best path in the term of metric and hop count between the source and destination. In the proposed segmental routing mechanism, the path calculation and management is divided in between the different network segments, where each of the segment compute its path until the last node in the segment in the specific direction[10]. The network path is managed across each of the segment among the path. Hence, whenever the path went down and the node failure is detected, the network path updation takes places in the segment with the node failure instead of computing the end-to-end path calculation [15]. The consideration of the path failure after the recalculation can't be ruled out; hence the probability of the path or connectivity failures is computed using the node availability assessment program. The following equation defines the method to compute the probability of path failures among the current path: $\mathrm{P}=$ (Total Availability Connection Failure $)^{2}$ Where total availability describes the number of occurrences of the current node in multiple paths, and the connection failure computes the total number of node failures occurred on this node till now. The following algorithm describes the overall 
working of the proposed segmental path routing algorithm:

Algorithm 1

(1) Deploy the network nodes in the given topology

(2) Perform the neighbor formation among the network nodes to create 1-hop connections

(3) Find the anchor nodes in the network with the highest degree of connectivity than its neighboring nodes

(4) Connect the cluster head nodes in the clusters of different segments with each other over the anchor nodes

(5) Initiate the segmental path routing module between the source and destination nodes

(6) Find the path from the source node to the last in the segment towards to destination node

(7) Run the node availability assessment agent (NA3) to assess the availability of the nodes on the path

a) Check if the node has NA3 available and running, then assign the node availability bit

b) Otherwise do not assign the node availability bit

(8) If 7(a)becomes true, then do the following steps:

a) Mark the node as path node

b) Add the node to the path vector

c) Update the segmental route data for the given segment

(9) Run the NA3 module between all nodes on the path on specific interval

(10)If any node returns NA3 failure

a) Verify the node availability again

b) Recognize the segment of the node failure

c) Perform steps 6 to 8 for the particular segment

\section{RESULT ANALYSIS}

1) The performance of the proposed model has been compared using the performance parameters of end-to-end delay and number of anchor nodes and energy consumption. The end-to-end delay (or transmission delay) parameter analyzes the overall time taken for the transmission of packets from source node to destination node. The number of anchor (aggregator) nodes indicates the intersectional points (routing nodes) across the selected path. The energy consumption parameter has been analyzed to understand the overall consumption of the energy in the proposed model simulation the work.

2) The proposed model simulation has been tested in 10 simulation rounds, where in each of the rounds, certain number of packets are retransmitted between the network nodes. The network performance has been tested with the pseudo randomly generated topology with 120 nodes. In figure 4 , The transmission delay in the proposed model has been observed between 0.0001 to 0.0006 seconds on all of the rounds. The average value of transmission delay has been computed nearly at 0.0004 seconds, which shows the significantly robust performance of the proposed model.

Fig. 4: The transmission delay obtained from the simulation
Fig. 5: Count of anchor nodes among the network path

In the figure 5, the number of anchor (aggregator) nodes is also observed in the network topology, which shows the quality of selected paths. The number of anchor nodes should not be less than $10 \%$ of the total network nodes, which indicates the poorly connected network and higher number of edge or leaf nodes. In this topology, 29.17\% nodes are found anchor nodes among the total network nodes. This ratio of anchor nodes shows the robustness and flexibility of the wireless mesh network topology.

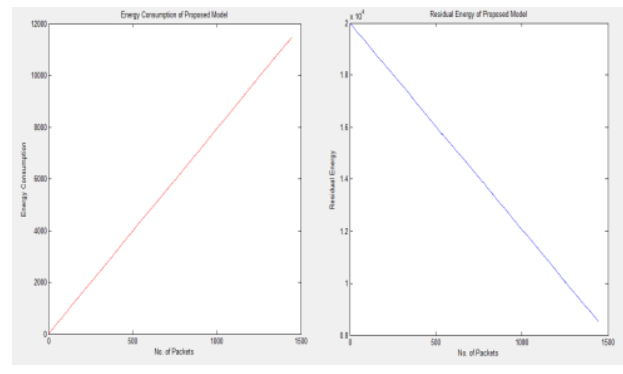

Fig. 6: (a) Energy consumption (b) Residual Energy

In figure 6, the energy consumption and residual energy has been analyzed in the proposed topology, where the similar ratio has been observed among both of the graphs in this figure. The energy consumption has been shown up to 12000 joules, and precisely it's been recorded between 11000 and 12000 joules for the transmission of 1500packets. The residual energy value shows the remaining energy slightly higher than 8000 joules, which is exactly inverted value of energy consumption.

Table I: Comparative Analysis of Proposed Model on the basis of Throughput

\begin{tabular}{|l|c|c|c|r|r|}
\hline & \multicolumn{5}{|c|}{ Average Throughput (Existing) } \\
\hline $\begin{array}{l}\text { Network } \\
\text { Scenario }\end{array}$ & LTE25 & LTE50 & Wi-Fi & CHR25 & CHR50 \\
\hline $\begin{array}{l}\text { Uplink 19 Nodes } \\
\text { Random }\end{array}$ & 366.23 & 570.2 & 1057.5 & 1734 & $\begin{array}{r}2134.1 \\
8\end{array}$ \\
\hline $\begin{array}{l}\text { Uplink 30 Nodes } \\
\text { Random }\end{array}$ & 355.59 & 562.3 & 679.52 & 1022.3 & $\begin{array}{r}1331.4 \\
8\end{array}$ \\
\hline $\begin{array}{l}\text { Downlink 19 } \\
\text { Nodes Random }\end{array}$ & 558.06 & 846.95 & 725.3 & 1644.2 & $\begin{array}{r}2233.4 \\
5\end{array}$ \\
\hline $\begin{array}{l}\text { Downlink 30 } \\
\text { Nodes Random }\end{array}$ & 346.48 & 524.24 & 471.54 & 1078.6 & 1557.9 \\
\hline & & \multicolumn{5}{|c|}{ Average Throughput (Proposed) } \\
\hline & LTE25 & \multicolumn{5}{|c|}{ LTE50 } & Wi-Fi & CHR25 & CHR50 \\
\hline
\end{tabular}

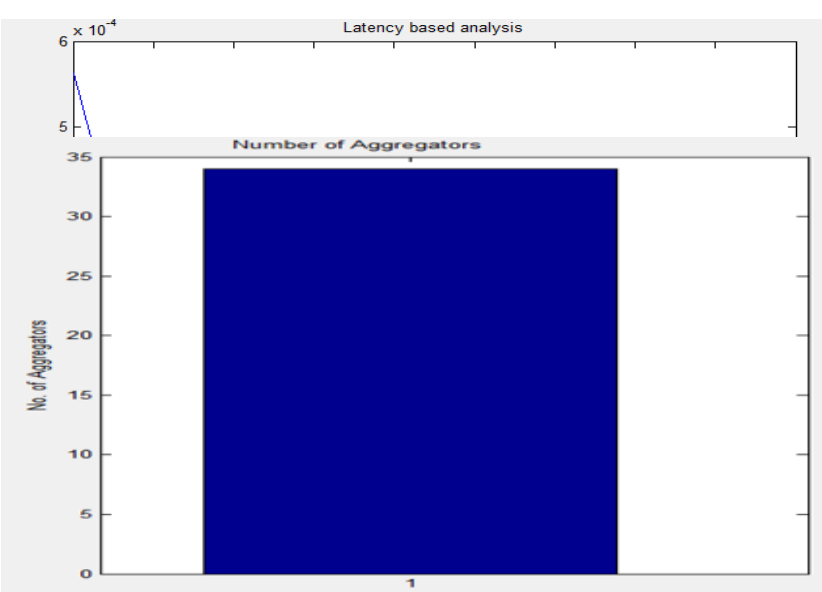




\begin{tabular}{|l|r|r|r|r|r|}
\hline $\begin{array}{l}\text { Uplink 19 Nodes } \\
\text { Random }\end{array}$ & 754.51 & 1497 & 2103.9 & 1859.3 & 2150.8 \\
7 & & 7 \\
\hline $\begin{array}{l}\text { Uplink 30 Nodes } \\
\text { Random }\end{array}$ & 748.91 & 1495.8 & 2098.8 & 1894.1 & 2160 \\
\hline Downlink 19 & 1494.1 & 3004.2 & 4200.0 & 3743.9 & 4287.8 \\
Nodes Random & 6 & 4 & 4 & 2 & 9 \\
\hline Downlink 30 & 1506.9 & 2992.0 & 4193.1 & 3796.1 & 4314.9 \\
Nodes Random & 1 & 7 & 8 & & 3 \\
\hline
\end{tabular}

The proposed model has been deeply analyzed for the throughput parameter in comparison with the existing model. The proposed model has been analyzed with combination of 19 and 30 randomly deployed nodes in the uplink and downlink scenarios. The proposed model has been designed to handle the overloaded volumes efficiently by using the load balancing mechanism. The proposed model has been recorded with higher throughput on all of the simulation scenarios in the table 1

Table II: Comparative analysis on the basis of average values

\begin{tabular}{|c|c|c|c|c|c|}
\hline $\begin{array}{c}\text { Average } \\
\text { Values }\end{array}$ & LTE25 & LTE50 & Wi-Fi & CHR25 & CHR50 \\
\hline Exising & 406.59 & 625.9225 & 733.465 & 1369.805 & 1814.258 \\
\hline Proposed & 1126.123 & 2247.293 & 3149.003 & 2823.358 & 3228.423 \\
\hline
\end{tabular}

In table 2, the proposed model has been recorded with 1126 Kbps against the $406 \mathrm{Kbps}$ in the existing model for the LTE25 models. For the Wi-Fi model, the proposed model has outperformed the exiting model by $2247 \mathrm{Kbps}$ in comparison with $626 \mathrm{Kbps}$ of existing model. The proposed model has been learnt to outperform the existing model in all of the tested models as observed from the proposed model results.

\section{V.CONCLUSION}

The proposed model has been designed with the segmental routing mechanism along with load balancing to handle the heavier amounts of data. The proposed model performance has been recorded in the form of throughput, energy consumption, transmission delay and number of anchor nodes. The proposed model has been recorded with 0.0004 seconds, which is considered significant value to show the high performance of the network cluster under proposed model. The proposed model has been recorded with nearly $29 \%$ of the nodes as anchors out of the total 120 nodes, which is quite higher than minimum requirement of $10 \%$, and signifies the well converged network in the proposed model simulation. In the proposed model the simulation has been recorded with the energy consumption of nearly 12000 joules for the transmission of 1600 packets. The proposed model has been recorded with 1126 and $2247 \mathrm{Kbps}$ for the LTE25 and LTE50 models against the existing model's 406 and $625 \mathrm{Kbps}$ respectively, whereast throughput value of 2823 and $3228 \mathrm{Kbps}$ for CHR25 and CHR50, which outperforms the existing mdoelwith 1370 and 1814 Kbps.

\section{REFERENCES}

1. Kwon, Kiwoong, Minkeun Ha, Taehong Kim, Seong Hoon Kim, and Daeyoung Kim. "The stateless point to point routing protocol based on shortcut tree routing algorithm for IP-WSN." In Internet of Things (IOT), 2012 3rd International Conference on the, 2012, pp. 167-174.

2. Bechkit, Walid, Mouloud Koudil, Yacine Challal, Abdelmadjid Bouabdallah, Brahim Souici, and Karima Benatchba. "A new weighted shortest path tree for convergecast traffic routing in WSN." In Computers and Communications (ISCC), 2012 IEEE Symposium on, 2012, pp. 187-192.

3. Delaney, D., Russell Higgs, and G. O'Hare. "A stable routing framework for tree-based routing structures in wsns." 2014, pp. 1-1.

4. Ghadimi, Euhanna, Olaf Landsiedel, Pablo Soldati, Simon Duquennoy, and Mikael Johansson. "Opportunistic Routing in Low Duty-Cycled Wireless Mesh networks." ACM Transactions on Mesh networks vol. 10(4), 2014.

5. Sahin, Dilan, Vehbi Cagri Gungor, Taskin Kocak, and Gurkan Tuna. "Quality-of-service differentiation in single-path and multi-path routing for wireless mesh network-based smart grid applications." Ad Hoc Networks, 2014.

6. Singh, Dharmendra, Shubhanjali Sharma, Vinesh Jain, and Jyoti Gajrani. "Energy efficient source based tree routing with time stamp in WSN." In Signal Propagation and Computer Technology (ICSPCT), 2014 International Conference on, 2014, pp. 120-124.

7. Tunca, Can, Sinan Isik, M. Donmez, and Cem Ersoy. "Distributed Mobile Sink Routing for Wireless Mesh networks: A Survey." 2014, pp. 1-21.

8. Chakraborty, Dibakar. "i-QCA: An intelligent framework for quality of service multicast routing in multichannel multiradio wireless mesh networks." Ad Hoc Networks vol. 33, 2015, pp. 221-232.

9. Lall, Shruti, B. T. J. Maharaj, and PA Jansen van Vuuren "Null-frequency jamming of a proactive routing protocol in wireless mesh networks." Journal of Network and Computer Applications, 2015 .

10. Lai, I-Wei, Wen-Chung Kao, and Nobuo Funabiki. "Cross-layer selective routing for active access-point minimization in wireless mesh network." InConsumer Electronics-Taiwan (ICCE-TW), 2015 IEEE International Conference on, 2015, pp. 482-483.

11. Al-Saadi, Ahmed, Rossitza Setchi, Yulia Hicks, and Stuart M. Allen. "Routing Protocol for Heterogeneous Wireless Mesh Networks." IEEE Transactions on Vehicular Technology, vol. 65 (12), 2016, pp. 9773-9786

12. Zhou, Anfu, Min Liu, Zhongcheng Li, and Eryk Dutkiewicz. "Joint Traffic Splitting, Rate Control, Routing, and Scheduling Algorithm for Maximizing Network Utility in Wireless Mesh Networks." IEEE Transactions on Vehicular Technology, vol. 65(4), 2016, pp. 2688-2702.

13. Murugeswari, R., S. Radhakrishnan, and D. Devaraj. "A multi-objective evolutionary algorithm based QoS routing in wireless mesh networks." Applied Soft Computing, vol. 40, 2016, pp. 517-525.

14. Sakamoto, Shinji, Tetsuya Oda, Makoto Ikeda, Leonard Barolli, and Fatos Xhafa. "Implementation of a New Replacement Method in WMN-PSO Simulation System and Its Performance Evaluation." In Advanced Information Networking and Applications (AINA), 2016 IEEE 30th International Conference on, 2016, pp. 206-211.

15. Hassan, Sharma, Singh "Detection and Mitigation of Selfish Node in Wireless Mesh Networks (WMN's)" international journal of technology and computing (ijtc), ISSN-2455-099X, vol. 5(5), 2019.

\section{AUTHORS PROFILE}

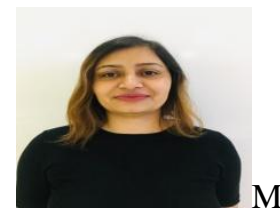

Manmeet Kaur is currently working as an Assistant Professor at Chandigarh Group of Colleges, Landran, Mohali, India..She completed her MTech from CDAC Mohali, Punjab , and B.Tech from AIET, Faridkot,Punjab India. She has published more than 8 
research papers in reputed National and International Conferences and Journals. She has Filed 8 Patents \& having more than 7 years of academic experience

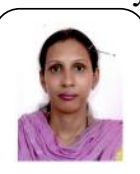

Preeti Bansal is currently working as an Assistant Professor at Chandigarh Group of Colleges, Landran, Mohali, India..She completed her M.Tech from PTU Jalandhar Punjab , and B.Tech from HCE Sonepat Haryana India. She has published more than 10 research papers in reputed National and International Conferences and Journals. She has Filed 6 Patents \& having more than 13 years of academic experience.

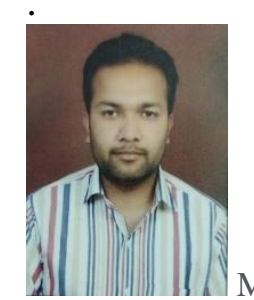

Mr. Gaurav Kumar, M.Tech(ECE), is an Assistant Professor and Researcher at the department of Electronics \& Communication Engineering, Chandigarh Engineering College, Landran. He worked on various projects and Patents for last 10 years. He has done his Masters in Engineering from GNDEC, Ludhiana. His area of interest is Communication Engineering. 